\title{
Frank Rösch: Nuclear and Radiochemistry, Volume 1: Introduction
}

\author{
W. De Gruyter Textbook, Berlin, 2014. ISBN 978-3-11-022191-6
}

\author{
Marion de Jong
}

Published online: 24 March 2015

(C) Springer-Verlag Berlin Heidelberg 2015

In this book, you can find "everything you always wanted to know about nuclear chemistry and radiochemistry, but were afraid to ask".

Nuclear chemistry is a vital field of basic and applied research. Nuclear chemistry and radiopharmaceutical chemistry are increasingly used as the bridge from pharmaceutical and medical research to state-of-the-art noninvasive molecular imaging as well as to patient-tailored treatment.

Based on state-of-the-art information, this book teaches the fundamentals of nuclear chemistry. It gives an introduction to the important field of nuclear chemistry for graduate students and post-docs working in the field of nuclear chemistry. In addition, it may serve as a great source of information for all working and/or interested in the broad field of nuclear medicine and nuclear medicine research, including nuclear medicine physicians, physicists, pharmacists, technicians, biologists and biochemists.

The current Volume 1 contains 14 chapters and 471 pages, it is richly illustrated with 285 figures and it contains 83 tables. In the various chapters the author addresses the structure of atoms and the nuclei of atoms, the transformation of unstable nuclei to more stable nucleon configurations, the mechanisms of the main transformation pathways and their kinetics, the character of the radiation emitted from these processes, the interaction of this radiation with condensed matter, and finally nuclear reaction processes to produce new nuclei.

The book may serve as a clear guide to understanding the essence of radioactivity, answering many questions such as: Why is the nucleus of an atom stable or unstable? What stimulates an unstable nucleus to transform? What are the velocities of the transformations? What are the main properties of the various emissions released in the course of a transformation? How can some of the particularly important radionuclides be produced artificially?

The complete text is to be in two volumes, with Volume 2 "Modern Applications" expected in May 2015. This part will deal with modern applications of nuclear chemistry concerning various scientific disciplines, such as highly sensitive, highly selective and nondestructive analytical technologies, pharmaceutical and medical research with state-of-the-art noninvasive molecular diagnosis, and with individualized patient treatment and nuclear energy. This book also discusses the issues of waste management and environmental aspects.

This book fulfils well the aims of the author. Volume 1 is very clearly written, gives a lot of information on the topics mentioned and is highly recommended for the different reader groups as described.

M. de Jong $(\bowtie)$

Erasmus MC, Rotterdam, The Netherlands

e-mail: m.hendriks-dejong@erasmusmc.nl 